\title{
Molecular Dynamic Simulation Analysis on Marine Fungi Compounds Against EGFR and VEGFR-2 Inhibitory Activity in Non-Small Cell Lung Cancer
}

\author{
Arry Yanuar, Kinanti Khansa Chavarina, Rezi Riadhi Syahdi* \\ Faculty of Pharmacy, Universitas Indonesia, Depok, INDONESIA.
}

\begin{abstract}
Introduction: According to International Agency for Research on Cancer (IARC), the number of lung cancer patients has reached 1.8 million lives, and $85 \%$ of the number contribute to non-small cell lung cancer. In the past years, research on targeted therapy has been developed due to its efficacy and a small number of side effects. Research on marine fungi compounds has not been explored to non-small cell lung cancer therapy. Methods: This research uses molecular dynamics simulation method to marine fungi compounds that have been docked to EGFR (FU0015, FU0051, FU0202) and VEGFR-2 (FU0033) as antiproliferative and antiangiogenetic agent by inhibition activity using AutoDock and AMBER at $300 \mathrm{~K}$ and $310 \mathrm{~K}$ temperature using EGFR (Gefitinib, Erlotinib, and Imatinib) and VEGFR-2 (Nicotinamide and Vatalanib) as reference standards. Results: Molecular dynamics results for EGFR inhibitors at 310K shows the best MMGBSA free energy and hydrogen occupancy in FU0051 (-43.72 kcal/mol; $98.80 \%)$ followed by FU0202 (-31.64 kcal/mol; 43.35\%), and FU0015 (-15.55 kcal $/ \mathrm{mol} ; 3.35 \%)$. FU0033 fungi as a material for VEGFR-2 inhibitor shows higher MMGBSA
\end{abstract}

free energy in comparison to its reference standards and low hydrogen occupancy $(0.15 \%)$ at $310 \mathrm{~K}$. Conclusion: This research shows that FU0051 and FU0202 have potential to be an antiproliferative agent candidate, hence in vitro test should be obtained.

Key words: EGFR, Lung cancer, Marine fungi compounds, Molecular dynamics, VEGFR-2.

\section{Correspondence}

Rezi Riadhi Syahdi, Faculty of Pharmacy, Universitas Indonesia, Depok, 16424 INDONESIA.

Phone: +6287878588772

Email: rezi.riadhi@farmasi.ui.ac.id, icapps2017.farmasiui@gmail.com DOI: 10.5530/jyp.2018.2s.6

\section{INTRODUCTION}

Patients with lung cancer have reached 1.8 million people in the world. ${ }^{1}$ As many as $85 \%$ of these figures are non-small cell carcinoma lung cancer and is predicted to continue growing by 200 thousand inhabitants with the number of deaths of 160 thousand in $2030 .{ }^{2}$ Currently there are many treatment options, but still, have many limitations especially on side effects and resistance. ${ }^{3}$ Many research to support the discovery of new therapies has been done elsewhere.

Exploration of new compounds to seek effective treatment has been largely done with several therapeutic approaches, one targeted therapy. Research on targeted therapies is being developed as it proves to have more effective therapeutic outcomes and fewer side effects. ${ }^{4}$ One example of therapies targeted use in lung cancer types carcinoma small cell is an inhibitor of Epidermal Growth Factor Receptor (EGFR) and Vascular Endothelial Growth Factor Receptor-2 (VEGFR-2) which inhibits the proliferation and survival of cancer cells. ${ }^{5}$

EGFR and VEGFR-2 are receptors that signal through the binding of tyrosine kinases and contribute to the regulation of cancer cell growth, migration, and apoptosis. ${ }^{6}$ Compounds inhibitor of EGFR and VEGFR-2 as Gefitinib and Vatalanib has been proven effective in cancer therapy and developed, but the exploration of new compounds made from nature (plants, animals, and microorganisms) that have the potential to the inhibition of EGFR and VEGFR-2 is still very small. ${ }^{7}$ The development of a chemical compound of natural ingredients serving as an anticancer has been supported by the National Cancer Institute (NCI) with a computational drug-based discovery and screening program. ${ }^{8}$
Screening methods Virtual is one of the methods in silico that are widely used to identify compounds new potential quickly, where this technique to cover the shortfall of the method in vivo which takes a long time and are relatively expensive. ${ }^{9}$ This method can reduce the number of compounds to be tested to give effect to certain proteins so as to obtain the compounds that have the highest activity for new drug candidates. Another commonly used method of silicon is the molecular dynamics simulation. Simulation of molecular dynamics can support the development of virtual screening compounds by showing the molecular interactions of atoms of active compounds in the action environment over a period as data supporting the ability of these compounds to provide their activity. ${ }^{10}$

In the study conducted by Watty (2017), finds that there are several types of marine fungi that have inhibitory activity against EGFR and VEGFR-2 uses virtual screening methods and obtained three active compounds which act as inhibitors of EGFR and an active compound which acts as an inhibitor of VEGFR- 2 of the Ascomycota phyla. ${ }^{11}$ However, these studies are limited to belay molecular without any analysis of the stability of the bond to the space and time that is necessary to analyze simulations of molecular dynamics of the activity of the compound inhibitors of the EGFR and VEGFR-2 of 4 biota fungi marine use parameter value Root Mean Square Deviation (RMSD), Root Mean Square Fluctuation (RMSF), hydrogen bonding conditions, and MMGBSA / MMPBSA in both vacuum and dissolved conditions at a specific temperature. ${ }^{12}$

This study will use four fungi marine biota previous research results, namely Fiscalin A, Aspergiolide B, and Sporothrin A as an EGFR inhibitor and Dankasterone A as a VEGFR-2 inhibitor. Reference standards are Gefitinib, Imatinib, and Erlotinib for EGFR inhibitors and have been 
widely used as anti-cancer, especially for patients with lung cancer as well as Nicotinamide and Vatalanib for inhibitors of VEGFR-2, which has passed through clinical trials phase $3 .^{13}$ The target macromolecules of EGFR and VEGFR-2 receptors are used only in the tyrosine kinase binding section of the Protein Data Bank site.

\section{MATERIALS AND METHODS}

\section{Materials \\ Hardware}

A single computer was used to conduct molecular docking and molecular dynamics simulations. The specification of the hardware and software are Intel ${ }^{\oplus}$ Xeon E5620 (Intel ${ }^{\oplus}$ Core $^{\mathrm{Tx}}$, American) processor (CPU) processors, Nvidia ${ }^{\circledR}$ GeForce GTX 780 (Nvidia, American) graphics processing unit (GPU) and 32 gigabytes (GB) Random Access Memory (RAM) DDR3. The computer ran the Linux operating system Ubuntu 12.04 LTS.

\section{Software}

AutoDock was used to carry out the molecular docking, while AMBER was used to conduct molecular dynamics simulations. LigandScout 4.09.2, LigPlot, and VMD were also used to visualize the ligand binding from molecular docking and molecular dynamics simulation results.

\section{Three-dimensional structure of EGFR and VEGFR-2}

The three-dimensional structure of EGFR and VEGFR-2 were obtained from the Research Collaboratory for Structural Bioinformatics Protein Data Bank (RCSB PDB) under identity name (ID) 4WKQ and 2P2I. Both structures were in complex with each ligand, Gefitinib for EGFR and Nicotinamide for VEGFR-2. Moreover, the structure was optimized by removing the water molecules and undesired molecules, adding polar hydrogens and Gasteiger charges.

\section{Three-dimensional structure of ligands}

The three-dimensional structure of ligands used in this study was Aspergiolide B, Dankasterone A, Fiscalin A, and Sporothrin A. Gefitinib, Erlotinib, and Imatinib was also used as reference standards of free energy binding value and ligand interactions of EGFR inhibitors and Nicotinamide and Vatalanib were also used for the same purpose of VEGFR-2 inhibitors. All these ligands were obtained from http://www. pubchem.com and http://chemspider.com. Charges were added to each ligand using AM1-BCC of Antechamber. Furthermore, all ligands were minimized using Sander to establish the relaxed structures. At last, all ligands were converted into pdbqt formats using python before performing the molecular docking.

\section{Methods}

\section{Molecular Docking}

Molecular docking was conducted using the AutoDock4 force field to generate the grid parameter files ${ }^{*}$.gpf) of ligand-ACE complexes. The grid box size used was configured into 60x60x60 with 0,375 Å spacing for EGFR and 50x50x50 with 0,375 $\AA$ spacing for VEGFR-2. The molecular docking was performed for 100 times (ga_run = 100). Scoring of free energy binding $(\Delta G)$ values of each ligand was performed based on the best energy and best cluster criteria. Furthermore, the visualization of ligand-ACE interactions was performed using LigandScout 4.09.2 and LigPlot. The interactions of ligand complexes were analyzed based on amino acid residues which were compared with respective reference standards.

\section{Molecular Dynamics Simulations}

Molecular dynamics simulations were carried out at temperatures of $300 \mathrm{~K}$ and $310 \mathrm{~K}$ within $20 \mathrm{~ns}$ for each ligand-macromolecule complex. The temperature of $300 \mathrm{~K}$ was chosen as a default temperature of standard molecular dynamics simulations, while the temperature of $310 \mathrm{~K}$ was selected as an attempt to follow the heat of a healthy human body., Each complex was analyzed on RMSD, RMSF, the hydrogen bond occupancy, free energy binding $(\Delta \mathrm{G})$ calculations using MM-GBSA and MM-PBSA methods.

\section{RESULTS}

\section{Molecular Docking}

Free energy binding $(\Delta G)$ are calculated using AutoDock software to check each ligand's affinity in compare to reference standards (Table 1). It shows that FU0015, FU0051, and FU0202 exhibited free energy binding $(\Delta \mathrm{G})$ values relatively lower than the free energy binding $(\Delta \mathrm{G})$ of reference standards for EGFR ligands.

\section{Molecular Dynamics Simulations}

Ligands, reference standards, and macromolecules of complex formations of molecular tethering were separated by the AutoDock program resulting bond stability analysis is measured in several parameters in two temperatures, $300 \mathrm{~K}$ and $310 \mathrm{~K}$, including:

\section{RMSD (root mean square deviation)}

Based on Figure 1, the RMSD value of the ligand tends to be more stable than the reference standard at $300 \mathrm{~K}$. The stability of fluctuations in FU0051 and FU0202 fungi ligand is seen in the 200th frame at 1.5-2 values. In contrast to the FU0015 ligand whose fluctuating stability

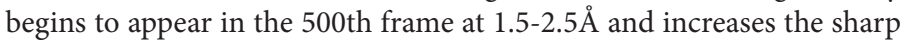
RMSD starting at the 1870th frame.

At $310 \mathrm{~K}$, RMSD ligand values also tend to be more stable than reference standards. FU0015 fungi ligand has a wide range of fluctuation stability. Initial temperature stability begins at the 719th to the 1395th frame with a fluctuation range between 1.3-2 $\AA$ and then stabilized again at the 1544 th to the 2000th frame with a fluctuation range of 2,3-3,1 $\AA$. FU0051 fungi ligand shows the stability of fluctuation starting from frame 681

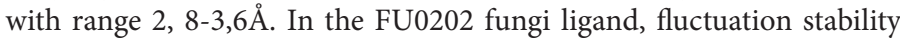
begins at 110th frame with a range of 1.7-2.4A.

In Figure 2, fluctuations in the RMSD value of the fungi ligand at a temperature of $300 \mathrm{~K}$ are much higher than the reference standard. The FU0033 fungi ligand shows an increase in the RMSD fluctuation value

\begin{tabular}{|ccccc}
\hline \multicolumn{3}{|c}{ Table 1: Free energy binding $(\boldsymbol{\Delta G})$ calculations results using AutoDock4. } \\
\hline Macromolecule & $\begin{array}{c}\text { Ligand } \\
\text { Code }\end{array}$ & Ligand Name & $\begin{array}{c}\text { Free Energy } \\
\text { / } \mathbf{\Delta G} \\
(\mathbf{k c a l} / \mathbf{m o l})\end{array}$ & $\begin{array}{c}\text { Inhibition } \\
\text { Constant } \\
(\mathbf{n M})\end{array}$ \\
\hline \multirow{2}{*}{ EGFR } & FU0015 & Fiscalin A & -7.59 & 2.72 \\
& FU0051 & Aspergiolide B & -8.91 & 296.42 \\
& FU0202 & Sporothrin A & -8.66 & 451.55 \\
& Reference & Gefitinib & -8.43 & 664.38 \\
& standards & Imatinib & -10.31 & 27.91 \\
& & Erlotinib & -7.23 & 4.99 \\
VEGFR-2 & FU0033 & Dankasterone & -11.43 & 4.20 \\
& & A & & \\
& Reference & Nicotinamide & -11.66 & 2.84 \\
& Standards & Vatalanib & -12.19 & 1.17 \\
\hline
\end{tabular}



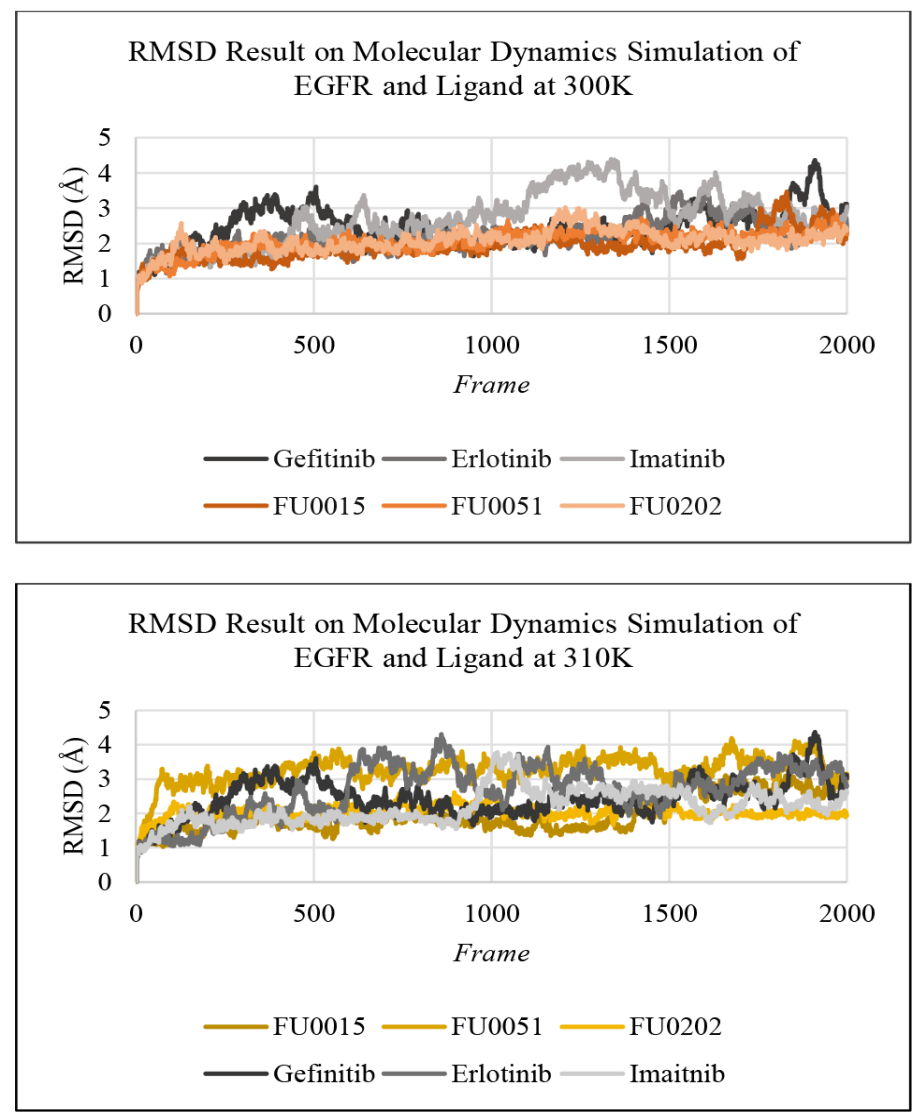

Figure 1: RMSD value of molecular dynamics simulation EGFR macromolecule in $20 \mathrm{~ns}$ at $300 \mathrm{~K}$ and $310 \mathrm{~K}$.

at the 321st frame and continues to rise until it reaches a $4 \AA$ peak on the 1203rd frame. The decrease in RMSD values starts at the 1700th frame and experiences a fluctuating stability of 2.5-3.

At $310 \mathrm{~K}$ temperatures, fluctuations in the RMSD value of FU0033 fungi ligand are more stable than the reference standards. The stability of RMSD fluctuations starts at the $81^{\text {st }}$ frame in the range of $1.19-2.1 \AA$.

\section{RMSF (root mean square fluctuation)}

According to Figure 3, the RMSF value at $300 \mathrm{~K}$ has the highest value occurring at amino acid residue number 749 worth $3,0301 \AA$. The ligand binding residue of THR 854, LEU 844, GLY 796, MET 793, LEU 792, THR 790, ALA 743, and LEU 718 showed very low RMSF values in all fungi ligands so that it could be categorized as having a more stable interaction with flexibility Low (Table 2 ). Similarly, at $310 \mathrm{~K}$, the average RMSF of the binding residue has a value lower than $1 \AA$, except for the FU0015 fungi ligand with LEU 718 residue having a value of $3.0407 \AA$. Differences in temperature parameters did not make a significant difference in the RMSF ligand and its reference standards.

The RMSF value of the target VEGFR-2 receptor in Figure 4 shows the peak value of the amino acid residue number 1011 with a value of $6.0211 \AA$ so that the amino acid is unstable and hardly binds. On the ligand binding sites of the amino acid GLU 917, VAL 916, LEU 889, GLU 885, LYS 868, ALA 866, VAL 848, and LEU 840 show very low RMSF values in FU0033 fungi ligands so that categorized interactions on active sites tend to be more Stable with low flexibility. At 310K temperatures, the RMSF of fungi FU0033 ligand did not give a significant difference, as shown in table 2.. Interactions on active sites remain low and tend to be stable.

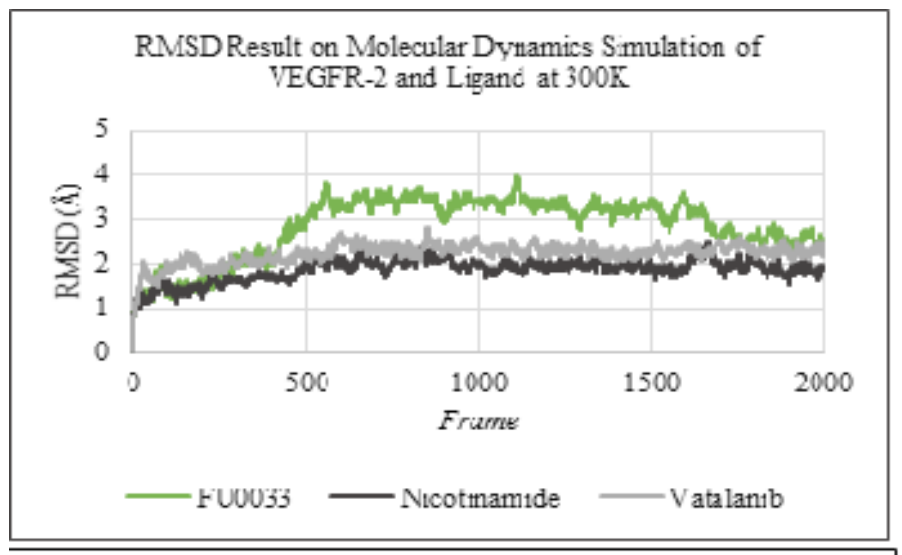

RMSI Result on Moleculır Dynanizs Simulation of VEGFK-2 andLigand at $310 K$

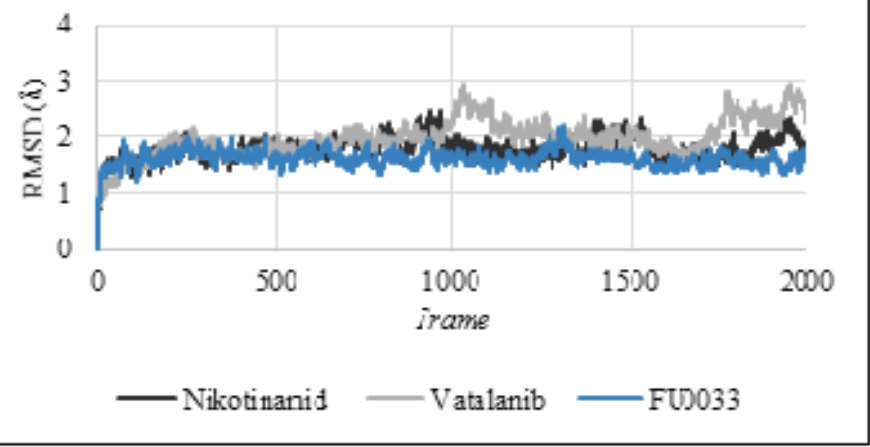

Figure 2: : RMSD value of molecular dynamics simulation VEGFR-2 macromolecule in $20 \mathrm{~ns}$ at $300 \mathrm{~K}$ and $310 \mathrm{~K}$.

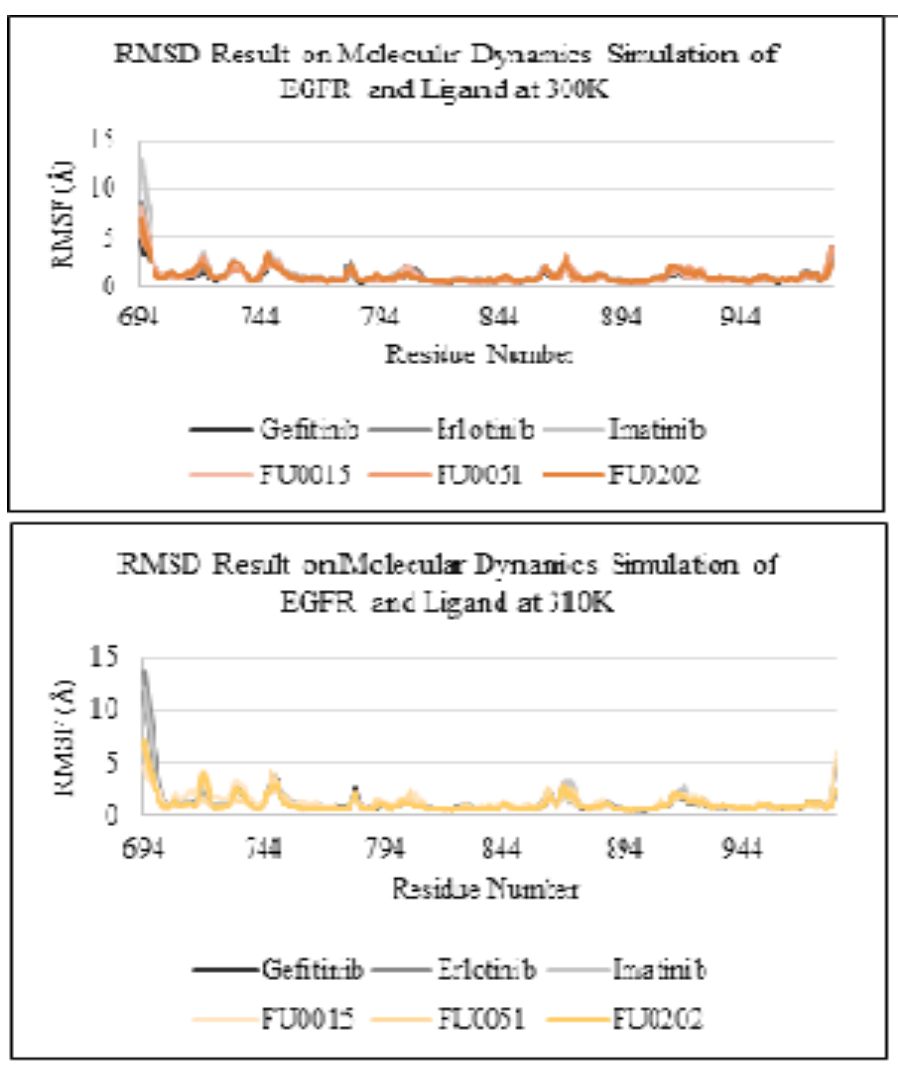

Figure 3: RMSF graphics of molecular dynamics simulation EGFR macromolecule in $20 \mathrm{~ns}$ at $300 \mathrm{~K}$ and $310 \mathrm{~K}$ 

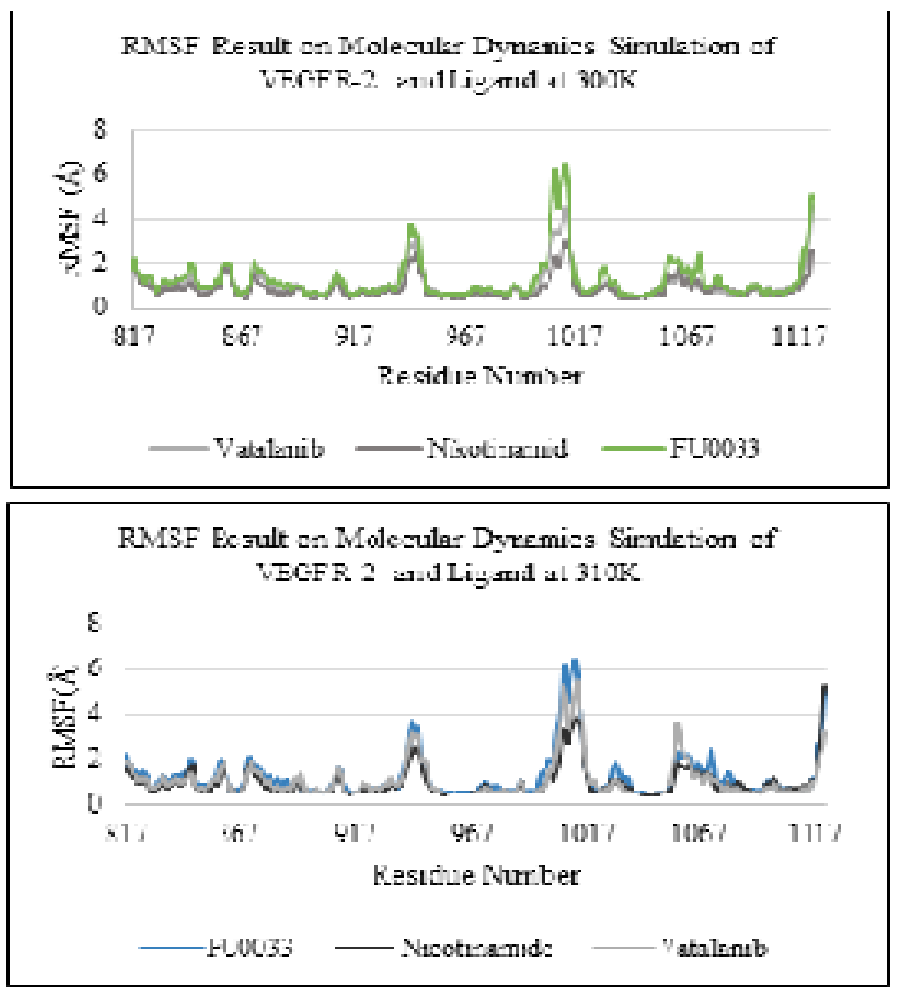

Figure 4: RMSF graphics of molecular dynamics simulation EGFR macromolecule in $20 \mathrm{~ns}$ at $300 \mathrm{~K}$ and $310 \mathrm{~K}$.

\section{The hydrogen bond occupancy}

The hydrogen bonding conditions of the molecular dynamics of the two receptor targets at a cutoff angle of $60^{\circ}$ with a distance of $3.0 \AA$ are dominated by electrostatic bonds (2.5-3.5 $\AA$ bond spacing). Based on Table 3 , the hydrogen bonding of FU0015 fungi ligand tend to be weak at $300 \mathrm{~K}$ indicated by the highest value of $20.95 \%$ in the amino acid residues of ASP 800 forming the hydrogen bond with the $\mathrm{O}$ chain of the side chain. While in the FU0051 fungi the highest occupancy rate of $72.25 \%$ occurred at the residue of ASP 855 with the $\mathrm{O}$ at the side chain followed by another bond on GLU 762 with the O side chain of $70.00 \%$. FU0202 highest occupancy value is at $5.50 \%$ in ASP 800 with the $\mathrm{O}$ atoms of the side chain, therefore that the hydrogen bonds can be categorized as weak. When compared to the reference standards, the hydrogen bond lever occupancy values tend to be the same or higher. Reference standard gefitinib found no hydrogen bond in the VMD program, while the reference standard of Erlotinib and Imatinib highest occupancy value was $36.95 \%$ and $3.85 \%$ respectively.

In Table 3, the hydrogen bond occupancy of the FU0015 fungi ligand at a temperature of $310 \mathrm{~K}$ is smaller than the previous temperature. The highest occupancy rate is only 3.35\% in SER 720 residue. Unlike FU0051 fungi ligand, the occupancy rate is much higher than the previous temperature. In the bond with residue ASP855, the occupancy rate reached $98.80 \%$ followed by bonding with GLU 762 with occupancy 97.95\% and THR 854 worth $67.05 \%$. In FU0202 fungi ligand, the highest bond value reaches $49.35 \%$ at MET793 residue. Reference standards show lower occupancy rates in Erlotinib but inversely proportional to Imatinib which has a value of up to $59.25 \%$ for the highest occupancy.

In Table 3, the FU0033 fungi ligand at 300K temperature shows a very low occupancy value of $0.25 \%$ at residual VAL 899 with the main chain

Table 2: RMSF value at ligand binding site with EGFR and VEGFR-2 macromolecule in $300 \mathrm{~K}$ and $310 \mathrm{~K}$ temperatures.

\begin{tabular}{|c|c|c|c|c|c|c|c|c|}
\hline \multirow{3}{*}{$\begin{array}{c}\text { Ligand Binding } \\
\text { Site } \\
\text { Temp. (K) }\end{array}$} & \multicolumn{8}{|c|}{ RMSF Value (Å) } \\
\hline & \multicolumn{2}{|c|}{ FU0033 } & \multicolumn{2}{|c|}{ FU0015 } & \multicolumn{2}{|c|}{ FU0051 } & \multicolumn{2}{|c|}{ FU0202 } \\
\hline & 300 & 310 & 300 & 310 & 300 & 310 & 300 & 310 \\
\hline CYS 919 & 0.5414 & 0.6762 & - & - & - & - & - & - \\
\hline GLU 917 & 0.5289 & 0.6193 & - & - & - & - & - & - \\
\hline VAL 916 & 0.4228 & 0.5316 & - & - & - & - & - & - \\
\hline LEU 889 & 0.6164 & 0.7968 & - & - & - & - & - & - \\
\hline GLU 885 & 0.5897 & 0.9636 & - & - & - & - & - & - \\
\hline LYS 868 & 0.4719 & 0.7117 & - & - & - & - & - & - \\
\hline ALA 866 & 0.5028 & 0.6518 & - & - & - & - & - & - \\
\hline VAL 848 & 0.5599 & 0.8794 & - & - & - & - & - & - \\
\hline LEU 840 & 0.9119 & 1.2624 & - & - & - & - & - & - \\
\hline THR 854 & - & - & 0.6528 & 0.9572 & 0.5678 & 0.5864 & 0.6528 & 0.6528 \\
\hline LEU 844 & - & - & 0.6622 & 1.1431 & 0.5897 & 0.7755 & 0.8791 & 0.7336 \\
\hline GLY 796 & - & - & 0.6723 & 0.9547 & 0.5778 & 0.7105 & 0.6858 & 0.8022 \\
\hline MET 793 & - & - & 0.7768 & 1.1924 & 0.6545 & 0.9361 & 0.8673 & 1.1129 \\
\hline LEU 792 & - & - & 0.7677 & 1.3800 & 0.5459 & 1.2535 & 0.8676 & 0.7419 \\
\hline THR 790 & - & - & 0.7299 & 0.8007 & 0.7838 & 0.7269 & 0.7549 & 0.5814 \\
\hline ALA 743 & - & - & 0.7813 & 1.1789 & 0.6604 & 0.5548 & 0.8380 & 06320 \\
\hline LEU 718 & - & - & 2.0102 & 3.0407 & 1.7055 & 1.0883 & 1.9529 & 2.0463 \\
\hline
\end{tabular}


Table 3: Hydrogen bonds occupancy of ligand and reference standards towards EGFR and VEGFR-2 macromolecule at 300K and 310K.

\begin{tabular}{|c|c|c|c|c|c|c|}
\hline Receptors & Temp. (K) & Code & Ligand Name & Donor & Acceptor & $\begin{array}{c}\text { Occupancy } \\
\text { (\%) }\end{array}$ \\
\hline \multirow[t]{3}{*}{ EGFR } & \multirow[t]{3}{*}{300} & \multirow[t]{3}{*}{ FU0015 } & \multirow[t]{3}{*}{ Fiscalin A } & LIG289-Side-N1 & ASP800-Side-OD1 & 20.95 \\
\hline & & & & CYS797-Main-N & LIG289-Side-O3 & 19.00 \\
\hline & & & & LIG289-Main-O & ASP800-Side-OD1 & 16.15 \\
\hline \multirow[t]{3}{*}{ EGFR } & \multirow{3}{*}{300} & \multirow[t]{3}{*}{ FU0051 } & \multirow[t]{3}{*}{ Aspergiolide B } & LIG289-Side-O7 & ASP855-Side-OD2 & 72.25 \\
\hline & & & & LIG289-Side-O8 & GLU762-Side-OE1 & 70.00 \\
\hline & & & & LIG289-Side-O8 & GLU762-Side-OE2 & 29.40 \\
\hline EGFR & 300 & FU0202 & Sporothrin A & LIG289-Side-O2 & ASP800-Side-OD2 & 5.50 \\
\hline \multirow[t]{6}{*}{ EGFR } & \multirow[t]{6}{*}{300} & \multirow{2}{*}{ Reference standards } & \multirow[t]{2}{*}{ Gefitinib } & MET793-Main-N & LIG289-Side-N2 & 13.30 \\
\hline & & & & LIG289-Side-C15 & GLN791-Main-O & 1.15 \\
\hline & & \multirow{3}{*}{ Reference standards } & \multirow[t]{3}{*}{ Erlotinib } & LIG289-Main-N & GLU797-Side-OE1 & 36.95 \\
\hline & & & & LYS745-Side-NZ & LIG289-Side-N2 & 36.95 \\
\hline & & & & THR854-Side-OG1 & LIG289-Main-O & 27.20 \\
\hline & & Reference standards & Imatinib & LIG289-Side-N1 & ASP800-Side-OD1 & 3.85 \\
\hline \multirow[t]{3}{*}{ EGFR } & \multirow[t]{3}{*}{310} & \multirow[t]{3}{*}{ FU0015 } & \multirow[t]{3}{*}{ Fiscalin A } & LIG289-Side-O1 & SER720-Side-OG & 3.35 \\
\hline & & & & CYS797-Main-N & LIG289-Main-O & 1.10 \\
\hline & & & & LIG289-Side-O1 & SER720-Main-N & 1.05 \\
\hline \multirow[t]{3}{*}{ EGFR } & \multirow[t]{3}{*}{310} & \multirow{3}{*}{ FU0051 } & \multirow[t]{3}{*}{ Aspergiolide B } & LIG289-Side-O7 & ASP855-Side-OD2 & 98.80 \\
\hline & & & & LIG289-Side-O8 & GLU762-Side-OE2 & 97.95 \\
\hline & & & & LIG289-Side-O4 & THR854-Side-OG1 & 67.05 \\
\hline \multirow[t]{3}{*}{ EGFR } & 310 & FU0202 & Sporothrin A & LIG289-Side-O4 & MET793-Main-N & 49.35 \\
\hline & & & & LIG289-Side-O2 & ASP855-Side-OD2 & 33.75 \\
\hline & & & & LIG289-Side-O2 & ASP855-Side-CG & 27.90 \\
\hline EGFR & 310 & Reference standards & Gefitinib & MET793-Main-N & LIG289-Side-N2 & 20.80 \\
\hline EGFR & 310 & Reference standards & Erlotinib & LIG289-Side-O2 & MET793-Main-N & 31.80 \\
\hline & & & & CYS797-Main-N & LIG289-Side-O3 & 7.90 \\
\hline EGFR & 310 & Reference standards & Imatinib & CYS797-Main-N & LIG289-Main-O & 59.25 \\
\hline & & & & LIG289-Side-N2 & LEU718-Main-O & 39.25 \\
\hline & & & & LIG289-Side-N1 & ASP800-Side-OD1 & 26.20 \\
\hline VEGFR-2 & 300 & FU0033 & Dankasterone A & VAL899-Main-O & LIG307-Side-C17 & 0.25 \\
\hline & & & & ARG1027-Side-NH2 & LIG307-Side-O1 & 0.15 \\
\hline VEGFR-2 & 300 & & Nicotinamide & ASP1046-Main-N & LIG307-Side-O1 & 51.45 \\
\hline & & Reference standards & & LIG307-Side-N1 & GLU885-Side-OE1 & 37.95 \\
\hline & & & & LIG307-Side-N1 & GLU885-Side-OE2 & 15.60 \\
\hline VEGFR-2 & 300 & Reference standards & Vatalanib & LIG307-Main-N & GLU885-Side-OE1 & 50.75 \\
\hline & & & & LIG307-Main-N & GLU885-Side-OE2 & 39.05 \\
\hline & & & & ASP996-Main-N & LIG307-Side-N2 & 21.15 \\
\hline VEGFR-2 & 310 & FU0033 & Dankasterone A & VAL899-Main-O & LIG307-Side-C17 & 0.25 \\
\hline & & & & ARG1027-Side-NH2 & LIG307-Side-O1 & 0.15 \\
\hline VEGFR-2 & 310 & & Nicotinamide & ASP1046-Main-N & LIG307-Side-O1 & 51.45 \\
\hline & & Reference standards & & LIG307-Side-N1 & GLU885-Side-OE1 & 37.95 \\
\hline & & & & LIG307-Side-N1 & GLU885-Side-OE2 & 15.60 \\
\hline VEGFR-2 & 310 & Reference standards & Vatalanib & LIG307-Main-N & GLU885-Side-OE1 & 50.75 \\
\hline & & & & LIG307-Main-N & GLU885-Side-OE2 & 39.05 \\
\hline & & & & ASP996-Main-N & LIG307-Side-N2 & 21.15 \\
\hline
\end{tabular}


Yanuar, et al.: EGFR2 and VEGFR in silico Activity of Various Marine Fungi Compound

Table 4: Energy calculation result of MMGBSA/MMPBSA in EGFR and VEGFR macromolecule at 310K and 300K temperature.

\begin{tabular}{|c|c|c|c|c|c|}
\hline \multirow[t]{2}{*}{ Receptor } & \multirow[t]{2}{*}{ Code } & \multirow[t]{2}{*}{ Ligand Name } & \multirow[t]{2}{*}{ Temp. (K) } & \multicolumn{2}{|c|}{ Free energy / $\Delta \mathrm{G}(\mathrm{kkal} / \mathrm{mol})$} \\
\hline & & & & MMGBSA & MMPBSA \\
\hline \multirow[t]{12}{*}{ EGFR } & FU0015 & Fiscalin A & 300 & $-22,3392 \pm 2,4200$ & $-25,7729 \pm 3,2607$ \\
\hline & & & 310 & $-15,5460 \pm 2,0332$ & $-17,8692 \pm 2,3556$ \\
\hline & FU0051 & Aspergiolide B & 300 & $-40,3224 \pm 3,0635$ & $-37,7877 \pm 3,2928$ \\
\hline & & & 310 & $-43,7187 \pm 3,4748$ & $-38,8184 \pm 4,3112$ \\
\hline & FU0202 & Sporothrin A & 300 & $-13,8820 \pm 2,5258$ & $-15,4251 \pm 2,3812$ \\
\hline & & & 310 & $-31,6447 \pm 3,1338$ & $-24,8828 \pm 4,6144$ \\
\hline & Reference standards & Gefitinib & 300 & $-47,9292 \pm 2,5336$ & $-42,1217 \pm 3,0753$ \\
\hline & & & 310 & $-43.7952 \pm 2.2047$ & $-34,4771 \pm 2,9907$ \\
\hline & & Erlotinib & 300 & $-51,4286 \pm 2,4212$ & $-41,3595 \pm 2,9375$ \\
\hline & & & 310 & $-36,8132 \pm 3,9167$ & $-29,7053 \pm 3,7636$ \\
\hline & & Imatinib & 300 & $-23,9427 \pm 2,3285$ & $-14,3774 \pm 3,6125$ \\
\hline & & & 310 & $-35,1774 \pm 3,1105$ & $-32,6165 \pm 3,7539$ \\
\hline \multirow[t]{6}{*}{ VEGFR-2 } & FU0033 & Dankasterone A & 300 & $-45,1433 \pm 2,3264$ & $-35,9195 \pm 3,1853$ \\
\hline & & & 310 & $-47,8915 \pm 2,8615$ & $-36,0131 \pm 2,6635$ \\
\hline & Reference standards & Nicotinamide & 300 & $-58,6467 \pm 3,6396$ & $-46,0868 \pm 4,1232$ \\
\hline & & & 310 & $-58,0013 \pm 3,2058$ & $-47,1906 \pm 3,0897$ \\
\hline & & Vatalanib & 300 & $-56,0691 \pm 3,1056$ & $-44,6250 \pm 3,6038$ \\
\hline & & & 310 & $-56,7651 \pm 2,0427$ & $-45,3238 \pm 2,6417$ \\
\hline
\end{tabular}

$\mathrm{O}$ atoms. The occupancy rate at $310 \mathrm{~K}$ (table 3 ) shows a lower number, at $0.15 \%$. Nicotinamide reference standard with Vatalanib at $300 \mathrm{~K}$ respectively shows the highest occupancy value of $51.45 \%$ and $50.75 \%$ so that it can be categorized as a moderate hydrogen bond. While the second occupancy value of reference standard at $310 \mathrm{~K}$ temperature increased, $61.00 \%$ and $50.75 \%$ respectively for Nicotinamide and Vatalanib on GLU residual 885 .

\section{Free energy binding calculations $(\triangle \mathrm{G})$ using MM-GBSA and MMPBSA methods}

The MMGBSA / MMPBSA (Table 4) calculation results in FU0015 and FU0202 fungi ligands show higher values than the 3 reference standards. In contrast to FU0051 which has a value of free energy is lower than Imatinib. This does not rule out the possibility that the fungi ligand has inhibitory activity on the EGFR receptor target because the site of the occurrence of the hydrogen bond is equal to the reference standard.

MMGBSA / MMPBSA calculation results (Table 4) show that FU0033 or Dankasterone A fungi ligands have higher values compared to their 2 controls positively. This proves that the Fungi FU0033 ligand has a lower bond strength compared to its reference standard despite having the same active site. In all fungi ligands and their reference standard over each macromolecule, the largest contribution of free energy value is to the van der Waals free energy.

\section{DISCUSSION}

\section{Molecular Docking}

As shown in Table 1, the lower free energy binding $(\Delta G)$ values, the better affinity of ligand towards its receptor. In EGFR ligands, FU0015, FU0051, and FU0202, show a better affinity in compare to its reference standards. A better affinity also shown in VEGFR ligand, FU0033, with a slightly higher free binding energy in compare to its reference standards. This translates that each ligand can be processed to molecular dynamics simulation.

\section{Molecular Dynamics Simulations}

In the early stages of this preparation, the ligands and macromolecules that have been separated from the complex are used for the simulation of molecular dynamics. The ligand and the reference standard of the separation result are given additional hydrogen atoms using Open Babel software, AM1-BCC charge with Antechamber program, and the addition of water solvent using TIP3P with size $10 \AA$ accessed via PuTTY.

Performed equilibration process gradually, starting from the minimization energy adjustment, temperature $300 \mathrm{~K}$ and $310 \mathrm{~K}$, density, and final equilibration. Temperature differences are made to see the bond stability of the default system of molecular dynamics and body temperature. The equilibration process is then validated by executing commands via PuTTY. The molecular dynamics simulation run for $20 \mathrm{~ns}$ which are divided into 2000 frames.

Both EGFR and VEGFR ligands are processed in AMBER software to assess the activity of molecular dynamics simulations. Result for EGFR, RMSD shows that all ligands are relatively stable in compare to its reference standards, both in temperature $300 \mathrm{~K}$ and $310 \mathrm{~K}$. RMSF result shows that temperature differences did not make significant changes to the RMSF values which are relatively stable interaction indicated with relatively low values in each ligand binding site for both $300 \mathrm{~K}$ and $310 \mathrm{~K}$ temperature. Highest hydrogen binding occupancy are shown in FU0051 with $72,25 \%$ in $300 \mathrm{~K}$ and $98.80 \%$ in $310 \mathrm{~K}$ between LIG289 and O7. Another atom of EGFR, O8, is also attached to LIG289 with a high hydrogen value, which are $70,00 \%$ in $300 \mathrm{~K}$ and $97,95 \%$ in $310 \mathrm{~K}$. This is different than other two ligands, FU0015 and FU020, which has a contrast in value for each temperature. FU0051 tends to be more stable for its hydrogen occupancy. Free energy binding on MMGBSA/MMPBSA calculation shows the best value in FU0051 and FU0202. As for FU0015, the value for the free energy binding only shows a slightly lower value than Imatinib in $300 \mathrm{~K}$ temperature while in $310 \mathrm{~K}$ the free binding energy goes higher in compare to all reference standards. All four values determine that FU0051 and FU0202 has the most probable activity towards 
EGFR, as shown in their free binding energy which are lower than the reference standards. Important interactions at MET 793 residue in FU0051 and FU0202 marks an inhibition activity, additionally with THR 790 residue interaction with FU0051.

Result for VEGFR-2 ligand, FU0033, the RMSD value has more fluctuations in compare to its reference standard in $300 \mathrm{~K}$ than $310 \mathrm{~K}$. RMSF value shows a low number in the ligand binding site of its amino acids. Hydrogen bonding is very low at $0,25 \%$ in temperature $300 \mathrm{~K}$ and $310 \mathrm{~K}$. As for the MMGBSA/MMPBSA calculation, FU033 has a lower affinity in compare to its reference standards, added with no interaction with important residues in VEGFR-2 receptors. Therefore, FU0033 does not indicate a positive result for inhibiting the receptor.

\section{CONCLUSION}

The molecular dynamics simulation results show that the compounds that have an activity to the target of EGFR receptor as a whole are Aspergiolide B and Sporothrin A. Aspergiolide B which has passed the in vitro test has anticancer activity in pulmonary adenocarcinoma, showing the activity occurs due to EGFR inhibition.

The characteristics of the VEGFR-2 inhibitor from the reference standard bond analysis have (1) the heteroaromatic ring attached to the residue $\mathrm{N}$ residue of CYS 919 and (2) the hydrogen bond acceptor pairs binding to the residues of ASP 1046 and GLU 885.

\section{ACKNOWLEDGEMENT}

The authors wish to acknowledge the grants PITTA 2017 provided by the Directorate of Research and Community Engagement, Universitas Indonesia for this study. The funder had no role in study design, data collection, and analysis, decision to publish, or preparation of the manuscript. LigandScout 4.09.2 is licensed to Arry Yanuar, Universitas Indonesia.

\section{CONFLICT OF INTEREST}

The authors hereby declare that they have no affiliations with organization, project or entity with commercial purposes or financial interest or without financial interest in which the subject or materials addressed in the manuscript.

\section{ABBREVIATIONS}

IARC: International Agent for Research on Cancer; EGFR: Epidermal Growth Factor Receptor; VEGFR-2: Vascular Endothelial Growth Factor Receptor-2; NCI: United States National Cancer Institute; PDB: Protein Data Bank; FDA: United States Food and Drug Administration; CPU: Computer Processing Unit; GPU: Graphical Processing Unit; RMSD: Root mean square deviation; RMSF: Root mean square fluctuation; MMGBSA: Molecular mechanics - generalized Born surface area ; MMPBSA: Molecular mechanics - Poisson - Boltzmann Surface Area

\section{REFERENCES}

1. IARC. GLOBOCAN Cancer Fact Sheets: Lung Cancer Estimated Incidence, Mortality and Prevalence Worldwide in. 2012. Available from: http://globocan iarc.fr/old/FactSheets/cancers/lung-new.asp.

2. Rahib L, Smith BD, Aizenberg R, Rosenzweig AB, Fleshman JM, Matrisian LM Projecting Cancer Incidence and Deaths in 2030: The Unexpected Burden of Thyroid, Liver, and Pancreas Cancers in the United States. Cancer Research. 2014;74(11):2913-21.

3. Pape VF, et al. Design, synthesis and biological evaluation of thiosemicarbazones, hydrazinobenzothiazoles and arylhydrazones as anticancer agents with a potential to overcome multidrug resistance. European Journal of Medicinal Chemistry. 2016;117:335-54.

4. Brown C. An elusive cancer target. 2016;53(7):S106-8

5. Biasini $M$, et al. SWISS-MODEL: Modeling protein tertiary and quaternary structure using evolutionary information. Nucleic Acids Research. 2014; 42(W1):W252-8.

6. Yan Y, et al. 99mTc-HYNIC-MPG: A novel SPECT probe for targeting mutated EGFR. Bioorganic and Medicinal Chemistry Letters. 2015;25(7):1647-52.

7. Ciardiello F, Tortora G. EGFR Antagonists in Cancer Treatment. New England Journal of Medicine. 2008;358(11):1160-74.

8. NCCN. [Guideline] National Comprehensive Cancer Network, Non-Small Cell Lung Cancer. 2016. Available from: https://www.nccn.org/professionals/physician_gls/pdf/nscl.pdf

9. Lionta E, Spyrou G, Vassilatis DK, Cournia Z. Structure-based virtual screening for drug discovery: principles, applications and recent advances. Current Topics in Medicinal Chemistry. 2014;14(16):1923-38.

10. Borhani DW, Shaw DE. The future of molecular dynamics simulations in drug discovery. Journal of Computer-Aided Molecular Design. 2012;26(1):15-26.

11. Watty M, Syahdi RR, Yanuar A. Database compilation and virtual screening of secondary metabolites derived from marine fungi as epidermal growth factor receptor tyrosine kinase inhibitors. Asian J Pharm Clin Res. 2017;10:142-7.

12. Allen M. Introduction to Molecular Dynamics Simulation. United Kingdom: John von Neumann Institute for Computing. 2004;23:1-28.

13. Jost LM, et al. Metabolism and Disposition of Vatalanib (PTK787/ZK-222584) in Cancer Patients. Drug Metabolism and Disposition. 2006;34(11):1817-28.

Article History: Submission Date : 29-11-2017 ; Revised Date : 28-01-2018; Acceptance Date : 14-03-2018.

Cite this article: Yanuar A, Chavarina KK, Syahdi RR. Molecular Dynamic Simulation Analysis on Marine Fungi Compounds Against EGFR and VEGFR-2 Inhibitory Activity in Non-Small Cell Lung Cancer. J Young Pharm. 2018;10(2)Suppl:s25-s31. 